\title{
Analysis and Synthesis of Tonal Aircraft Noise Sources
}

\author{
Matthew P. Allen ${ }^{1}$ \\ Virginia Polytechnic Institute and State University, Blacksburg, VA 24060 \\ Stephen A. Rizzi ${ }^{2}$ \\ NASA Langley Research Center, Hampton, VA 23681 \\ Ricardo Burdisso ${ }^{3}$ \\ Virginia Polytechnic Institute and State University, Blacksburg, VA 24060 \\ and \\ Selen $\mathrm{Okcu}^{4}$ \\ National Institute of Aerospace, Hampton, VA 23666
}

Fixed and rotary wing aircraft operations can have a significant impact on communities in proximity to airports. Simulation of predicted aircraft flyover noise, paired with listening tests, is useful to noise reduction efforts since it allows direct annoyance evaluation of aircraft or operations currently in the design phase. This paper describes efforts to improve the realism of synthesized source noise by including short term fluctuations, specifically for inlet-radiated tones resulting from the fan stage of turbomachinery. It details analysis performed on an existing set of recorded turbofan data to isolate inlet-radiated tonal fan noise, then extract and model short term tonal fluctuations using the analytic signal. Methodologies for synthesizing time-variant tonal and broadband turbofan noise sources using measured fluctuations are also described. Finally, subjective listening test results are discussed which indicate that time-variant synthesized source noise is perceived to be very similar to recordings.

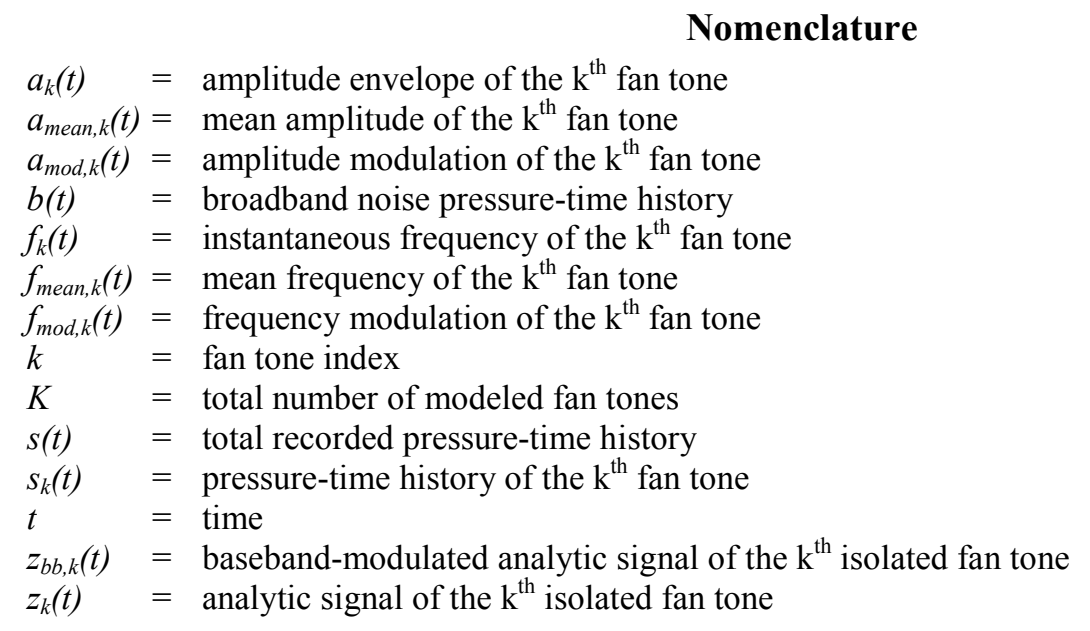

\footnotetext{
${ }^{1}$ Graduate Research Assistant, Mechanical Engineering Department, Student Member AIAA.

${ }^{2}$ Senior Research Engineer, Structural Acoustics Branch, Associate Fellow AIAA

${ }^{3}$ Professor, Mechanical Engineering Department, Member AIAA

${ }^{4}$ NIA Post-Doctoral Researcher
} 


\section{Introduction}

Fixed and rotary wing aircraft operations can have a significant impact on communities in proximity to airports. Trends in urbanization and increasing flight volume will serve to increase annoyance responses in the future, unless accompanied by noise reduction. One part of noise reduction efforts is the evaluation of the community noise impact of proposed aircraft during the design stages, which can serve as useful feedback for the manufacturer.

Annoyance evaluations are typically performed using one of three methods; field tests, laboratory presentation of recordings, or laboratory presentation of simulated flyover events. Synthesized flyover noise has advantages over field tests and recordings since it is much less expensive, allows more control over experimental variables, and can be used for unconventional aircraft and engine designs not in existence. Current simulation methods use predictionbased synthesis to generate source noise. Those predictions usually use time-invariant source noise parameters which are derived from long-term averages of measured data. However, noise sources are not completely characterized by time-invariant parameters, since they include short-term fluctuations which were lost by long-term averaging. Recent work has noted the lack of time variations in synthesized source noise that were observed in recordings ${ }^{1}$, while psychoacoustic literature shows that time-domain fluctuations in tonal parameters can be a significant discriminator for test subjects ${ }^{2}$. Available evidence suggested that short-term fluctuations in both tonal and broadband noise sources contributed to the perception of source noise realism, while the absence of fluctuations damaged that perception.

The main aim of the work presented in this paper is to improve the realism of simulated aircraft flyover noise, through improvements in realism of synthesized source noise. This was achieved in three ways. First, short term fluctuations were extracted from an existing set of turbofan engine recordings. For tonal noise, both the amplitude envelope and instantaneous frequency were of interest. The broadband noise source also contained short-term fluctuations. Tools were developed which used the complex-valued analytic signal to extract tonal parameters, and used the short-time Fourier transform to measure broadband fluctuations. A simple parametric model was developed to represent each measured tonal fluctuation based on its spectral bandwidth and variance. Second, synthesis tools capable of reproducing general time-variant tonal and broadband noise sources were developed. Inputs to the synthesis routines could either be generated according to the parametric model or directly supplied from measurements. The parameters of the model could be referenced to engine power setting and emission angle when synthesizing the fan noise source for arbitrary aircraft operations. Third, a psychoacoustic test was conducted to evaluate how the inclusion of short term fluctuations impacted the realism of the synthesized fan noise source. For that purpose, a set of subjective listening tests was conducted with members of the general public. Participants were asked to rate the similarity between noises synthesized according to time-invariant methods, time-variant methods, and actual engine recordings.

\section{Data Set}

The efforts to extract, analyze, and characterize short term fluctuations in fan noise were performed using an existing data set which was acquired in 2005 during joint NASA/Honeywell Aerospace tests called the Engine Validation of Noise and Emissions Reduction Technology (EVNERT) ${ }^{3}$. A demonstrator turbofan engine was used for several of the tests, under the designation TECH977. The TECH977 engine is a two-spool design in the business jet category, where the low-pressure shaft is designated as "N1" and the high-pressure shaft is designated "N2." The single stage fan (34.2" diameter, 22 blades), low-pressure compressor, and three-stage low pressure turbine (LPT) were all attached to the N1 shaft. The high-pressure compressor and corresponding two-stage high-pressure turbine were attached to the $\mathrm{N} 2$ shaft. Test conditions were originally intended to evaluate the noise reduction benefit of technologies such as inlet surface liners, relative to a baseline (unmodified) engine configuration. In particular, recordings of engine noise from the baseline configuration (number 19) were the focus of this investigation ${ }^{3}$.

As part of the test, the TECH977 demonstrator engine was mounted in a static test stand; a photograph of the setup can be seen in Figure 1. An inlet flow control device was installed to minimize turbulence ingested by the engine which might affect the radiated noise, and is visible in the photograph. For the baseline engine test configuration, a wooden barrier was constructed around the engine discharge to shield aft-radiated sources from reaching the microphone array. That barrier is not shown in Figure 1. In addition, there was no inlet acoustic liner installed in this baseline configuration. A diagram of the 32 microphone far-field array which was used to collect the data is shown in Figure 2. Synchronous pressure-time history recordings were made at five engine power settings; they were $48,54,60,71$, and $87 \%$ of the engine design point for maximum fan (N1) shaft rotation speed. Engine power settings of 48,54 , and $60 \%$ are referred to as "low" power settings, while 71 and $87 \%$ are referred to as "high" power settings. Each recording was sampled synchronously at each microphone at a rate of 32,768 samples per second for approximately 69.7 seconds. 


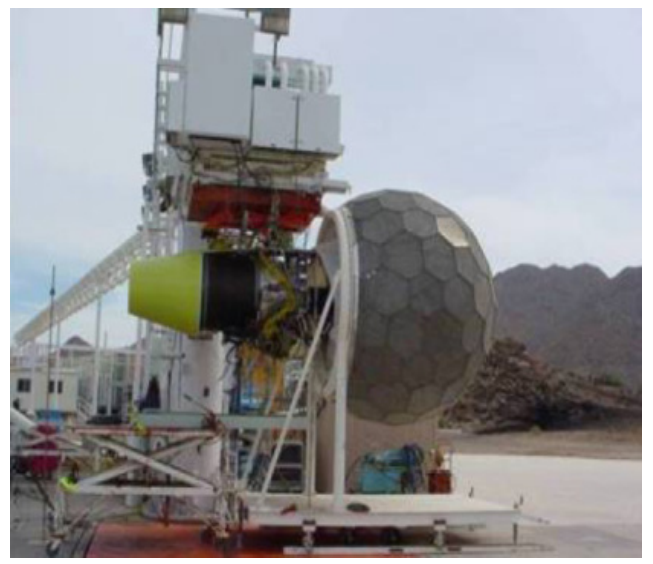

Figure 1. Photograph of the TECH977 engine mounted in the test stand. An inlet flow control device can be seen mounted around the engine inlet ${ }^{3}$.

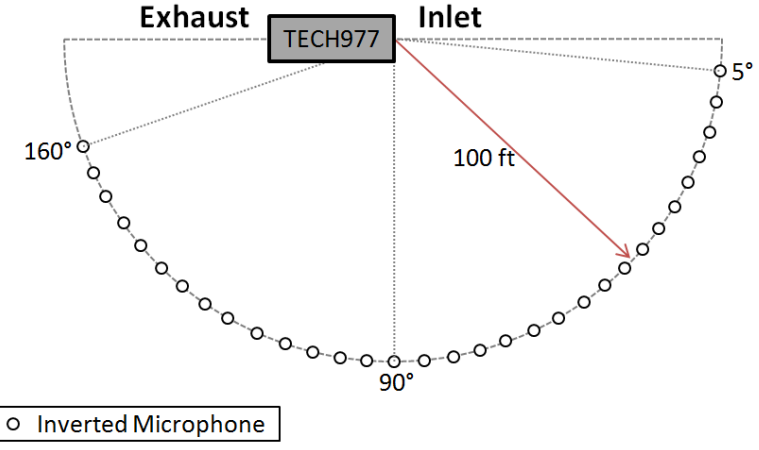

Figure 2. Diagram of the 32-microphone array used to capture recordings from the TECH977 engine.

Recorded spectra generally matched the expected characteristics of fan noise for both low and high power settings. A representative spectrum of the EVNERT recordings captured at low power settings can be seen in Figure 3, while a spectrum representative of high power settings is shown in Figure 4. The TECH977 engine noise displayed a significant tonal component at multiples of the blade passage frequency (BPF) at low fan power settings (blue), with an underlying broadband noise source. Also visible are tones resulting from compressor (pink) and lowpressure turbine (LPT) stages (orange), as well as from nonlinear interactions between the fan and high-pressure compressor (green). One interesting feature of recorded spectra is the presence of a significant broadband component peaking near $400 \mathrm{~Hz}$, most likely corresponding to the jet noise source transmitting through the barrier. Another feature evident from recorded spectra is the anti-aliasing filter used to ensure noise components above the acquisition Nyquist frequency were rejected. That filter can be seen to begin rolling off recorded spectra near 13,000 $\mathrm{Hz}$.

Recordings made at high power settings also contained multiple-pure-tone (MPT) noise due to fan blade tips which were supersonic (red). The MPT component was strong at angles radiating forward from the engine inlet (about $5^{\circ}$ to $50^{\circ}$ off-axis), and between angles of about $60^{\circ}$ and $100^{\circ}$ off-axis. Beyond that limit, all tonal noise was observed to roll off in amplitude. The spectrum shown in Figure 4 is representative of recordings captured at highpower settings where the MPT noise component was dominant. Shaft-order harmonics were observed over the entire recorded frequency range, though they decreased somewhat in amplitude as harmonic order increased. Broadband noise was also present in high-power recordings as expected, and a broadband noise peak near $400 \mathrm{~Hz}$ (likely due to jet noise) was also present. Special consideration was given to this region in all subsequent analysis, synthesis, and evaluation stages. For example, jet and combustion noise were filtered out of recorded noises during presentation to listeners in subjective evaluations. 


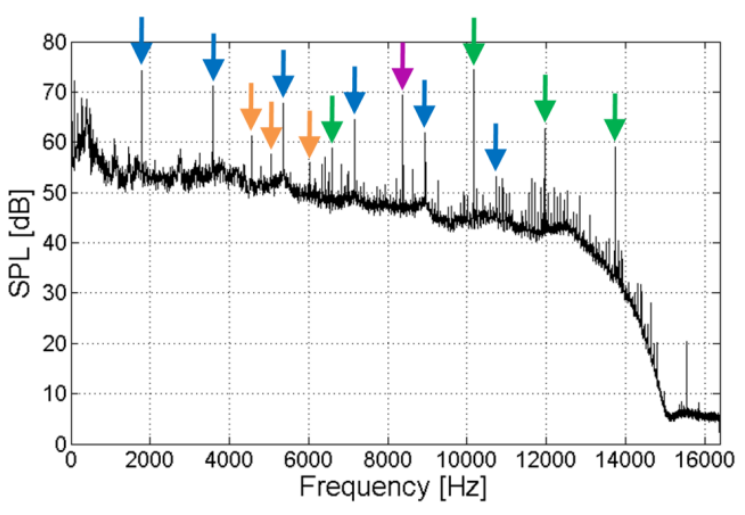

Figure 3. SPL spectrum of typical TECH977 recording at low power setting $\left(48 \%\right.$ power setting, $75^{\circ}$ emission angle).

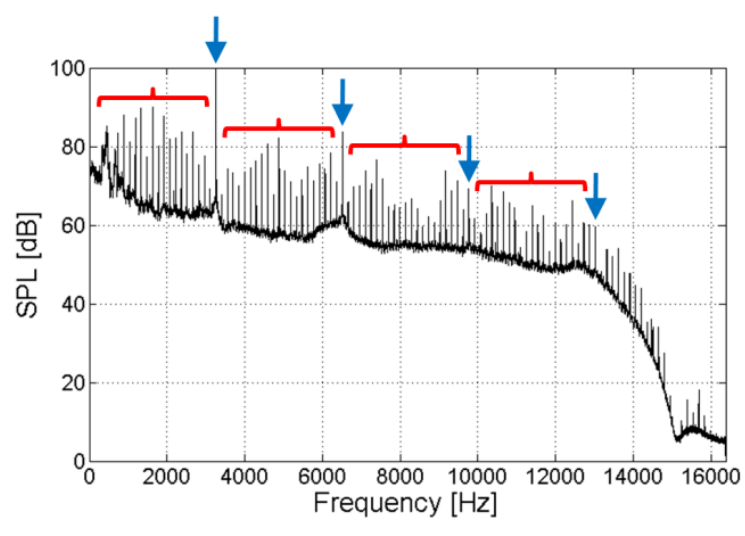

Figure 4. SPL spectrum of typical TECH977 recording at high power setting ( $87 \%$ power setting, $75^{\circ}$ emission angle).

The tonal analysis in the current investigation focused on inlet-radiated tones. Amplitude envelope and instantaneous frequency were not extracted for turbine, compressor, or fan/compressor interaction tones. However, identification of those tones was a useful exercise since their locations were used to refine other analyses. For example, the accuracy of the broadband analysis technique described later was improved by ignoring narrowband bins known to contain tonal components from turbine, compressor, or fan/compressor interaction mechanisms.

\section{Signals Analysis}

A simple representation of the signal recorded by each microphone at each power setting was created to facilitate analysis, and is shown for continuous time in Equation (1). Recorded noise consisted of a combination of tonal and broadband noise. The tonal source itself was a sum of many tones, arising from the combination of fan radiation sources described earlier. The representation for the total modeled signal was

$$
s(t)=\sum_{k=1}^{K} s_{k}(t)+b(t)
$$

where $s(t)$ is the total recorded source pressure-time history, $K$ is the total number of modeled fan tones, $s_{k}(t)$ is the pressure-time history of the $k^{\text {th }}$ tone, and $b(t)$ is time-variant broadband noise from several sources.

The time-variant broadband noise component $b(t)$ was estimated on a one-third-octave band basis. The method used to measure oscillations in one-third-octave band sound pressure levels (SPL) was derived from previous work which was geared toward the analysis and synthesis of jet noise sources ${ }^{1}$. Time-domain fluctuations in broadband noise spectra were extracted, but no model was generated for synthesis, as will be shown in the case of tonal noise. Instead, broadband noise was useful for creating a realistic analog of the total fan noise source during synthesis and subjective testing as described later.

\section{A. Model of Tonal Fluctuations}

To facilitate analysis of the combined tonal source, the pressure-time history of each component was represented as an amplitude and frequency-modulated cosine wave, as in

$$
s_{k}(t)=a_{k}(t) \cos \left(\phi_{k}(t)\right)
$$

where $a_{k}(t)$ is the amplitude envelope of the $k^{\text {th }}$ tone and $\phi_{k}(t)$ is the phase argument of the $k^{\text {th }}$ tone in radians. According to Equation (2), each tone was characterized completely by two parameters; the amplitude and phase functions. The relationship of the time-variant frequency of the cosine term to the phase argument in the single-tone model is described by

$$
\phi_{k}(t)=2 \pi \int_{-\infty}^{t} f_{k}(\tau) d \tau
$$

where $f_{k}$ is the instantaneous frequency of the $k^{\text {th }}$ tone in $\mathrm{Hz}$, and $\tau$ is a dummy variable of integration. Note that a tone of constant (time-invariant) frequency $f$ will have a phase integral which becomes the familiar $2 \pi f t$ argument of 
a simple harmonic oscillator. The instantaneous frequency may be obtained from Equation (3) by differentiating with respect to time as

$$
f_{k}(t)=\frac{1}{2 \pi} \frac{d\left(\phi_{k}(t)\right)}{d t} .
$$

Time variations in each of the cosine parameters (amplitude and frequency) were then represented as fluctuations around a time-invariant mean value according to

$$
\begin{aligned}
& a_{k}(t)=a_{\text {mean }, k}+a_{\text {mod }, k}(t) \\
& f_{k}(t)=f_{\text {mean }, k}+f_{\text {mod }, k}(t)
\end{aligned}
$$

where $a_{\text {mean }, k}$ is the mean amplitude of the $k^{\text {th }}$ tone, $a_{\text {mod, }}$ is the amplitude modulation of the $k^{\text {th }}$ tone around the mean value, $f_{\text {mean }, k}$ is the mean frequency of the $k^{\text {th }}$ tone, and $f_{\text {mod, } k}$ is the frequency modulation of the $k^{\text {th }}$ tone around the mean value.

\section{B. Demodulation using the Analytic Signal}

When considering various analysis methods for tonal noise, a procedure was desired which would provide high resolution in both the time and frequency domains. Further, the procedure must produce reliable, unique estimations for each tonal parameter. Time resolution was important to resolve fluctuations with bandwidth less than $20 \mathrm{~Hz}$, which are typically perceived as envelope or frequency variations rather than additional tonal components ${ }^{2}$. Additionally, high frequency resolution was desired to measure small deviations from the mean.

Previous attempts at tonal analysis had proceeded using the Short Time Fourier Transform (STFT) ${ }^{1}$. By identifying the frequency-domain location of a target tone at successive time steps, a record of variations in both frequency and tonal amplitude could be constructed. However, the STFT suffers from the inherent tradeoff between time and frequency resolution. That tradeoff was acceptable for broadband noise where a sum of many frequency bins was calculated to obtain the one-third-octave band representation. However, tones are highly localized in frequency, so high frequency resolution would be required to resolve them with confidence. However, that frequency resolution necessarily comes at the cost of decreased time-domain resolution, which serves to "smear" fluctuations of interest. For these reasons, a STFT analysis method was found unacceptable for tone tracking in the current investigation. An additional method utilizing a nonlinear fit of filtered data was also found to be unacceptable ${ }^{4}$. For this reason, an alternative analysis method was considered.

The method selected to extract the time-variant amplitude envelope and instantaneous frequency from tonal components of interest avoided the drawbacks of STFT analysis. The analysis methods for amplitude and frequency stem from techniques which are more commonly defined in the context of signal processing for communications. For example, in radio transmission, a modulation in either amplitude or frequency is applied to a carrier tone with a time-invariant mean amplitude and frequency. The problem of the receiver in that case is to separate the modulated carrier tone from the surrounding noisy environment, and then to separate the modulation itself from the carrier. The problem of extracting the amplitude envelope and instantaneous frequency of a particular tone was approached from that perspective, through construction of the analytic signal.

The analytic signal is a unique complex representation of any real-valued signal ${ }^{5-7}$. As a complex representation, it offers the benefit of direct calculation of the magnitude and phase angle at each signal sample. Three steps were applied to recordings to extract the amplitude envelope and instantaneous frequency for each tone of interest. First, each tone was isolated from other noise sources. Next, the "tone-isolated" signal was used to construct the complex-valued analytic signal. Finally, the magnitude and instantaneous frequency of the analytic signal were calculated to obtain the desired time domain fluctuations in tonal amplitude and frequency. Postprocessing used time-varying estimates of broadband noise to correct instantaneous frequency in regions of low signal-to-noise ratio (SNR).

The analytic signal may be constructed in several steps. First, the entire tone-isolated pressure-time history is transformed to the frequency domain. Next, all negative frequency bins are set to zero, and all positive frequency bins are multiplied by two. Finally, the signal is transformed back to the time domain, resulting in the complexvalued analytic signal.

Beginning with the single-tone parametric model described earlier, it can be $\operatorname{shown}^{4}$ that the corresponding analytic signal is

$$
z_{k}(t)=a_{k}(t) e^{j \phi_{k}(t)}
$$


where $z_{k}(t)$ is the complex-valued analytic signal, while $a_{k}(t)$ and $\phi_{k}(t)$ are the original amplitude and phase arguments. The problem of demodulation has now been transformed to one of calculating the instantaneous magnitude and phase of a single complex vector as a function of time.

Finding the magnitude of the complex quantity provides the amplitude envelope of the real-valued toneisolated signal, as in

$$
a_{k}(t)=\left|z_{k}(t)\right|=\sqrt{\operatorname{Re}\left(z_{k}(t)\right)^{2}+\operatorname{Im}\left(z_{k}(t)\right)^{2}}
$$

The mean value of the amplitude envelope is then removed to isolate the amplitude fluctuation of interest.

Next, the instantaneous frequency modulation is estimated. First, the analytic signal is modulated by a complex exponential at the opposite of the carrier frequency, which in this case is the N1 shaft frequency or one of its harmonics. This modulation has the effect of shifting the deviations to "baseband" (centered on zero frequency) according to

$$
\begin{aligned}
z_{b b, k}(t) & =z_{k}(t) \exp \left(-j 2 \pi f_{\text {mean }, k}\right) \\
& =a_{k}(t) \exp \left(j 2 \pi \int_{-\infty}^{t} f_{\text {mod }, k}(\tau) d \tau\right)
\end{aligned}
$$

which represents the complex-valued baseband analytic signal $z_{b b, k}(t)$ of the $k^{\text {th }}$ tone, and has instantaneous frequency equal to the deviation from the original mean value. The instantaneous phase of the baseband-modulated tonal signal is then calculated as

$$
\phi_{b b, k}(t)=\tan ^{-1}\left(z_{b b, k}(t)\right)
$$

Finally, the instantaneous frequency is found by the time derivative of the phase angle. Division by $2 \pi$ converts the angular frequency in radians per second to units of cycles per second, or Hz. So, the final representation for instantaneous frequency fluctuation is

$$
f_{\text {mod, },}(t)=\frac{1}{2 \pi} \frac{d}{d t}\left(\phi_{b b, k}(t)\right)
$$

where $f_{\text {mod,k }}(t)$ is calculated as the time derivative of the phase angle of the baseband-modulated analytic signal for the $k^{\text {th }}$ tone, $z_{b b, k}(t)$. The analysis described above was performed on discrete time series data. Discretized versions of Eqns. (1)-(11) are provided in ref. 4.

\section{Extraction of Fluctuations}

A MATLAB tool was developed to obtain the amplitude envelope and instantaneous frequency of the toneisolated recordings by utilizing the analytic signal according to the methodology outlined above. Four multiples of the BPF tone were analyzed in each recording at the three low-power settings $(48,54$, and $60 \%)$. Since the fan blade tips were supersonic at high power settings (71 and 87\%), characterizations of MPT noise were made from measurements at those conditions. The highest shaft-order tone analyzed was the $88^{\text {th }}$ fan order, which also corresponded to the $4^{\text {th }}$ multiple of the BPF. Theoretically, fan order tones were predicted to continue at significant levels to even higher frequencies, but the anti-aliasing filter used in data acquisition limited the reliable frequency range to about $13,000 \mathrm{~Hz}$.

For each tone, the analysis steps were applied in the following way. First, a bandpass filter with a $15 \mathrm{~Hz}$ passband centered on the tone was designed and applied to the total source noise recording. The filter was a $3^{\text {rd }}$ order Butterworth infinite-impulse response filter design. Since Butterworth filters have maximally flat passband response, the impact of the tone isolation step was assumed to have minimal impact on the frequency content of the isolated signal and thus on the amplitude and frequency fluctuations of interest. Application of the filter transformed the recorded signal which contained several tones as well as broadband noise into a single-tone signal.

Figure 5 shows the tone-isolation process as represented in the frequency domain. The top section shows a signal containing tonal and broadband noise, the center shows the designed isolation filter, and the bottom shows the comparison of measurement with the tone-isolated result. Figure 6 shows the process in the time domain, where the top section is the original recording and the bottom is the tone-isolated pressure-time history. Note the different scales on the vertical axes, designed to highlight fluctuations in tonal amplitude. A significant amount of energy was removed from the recorded signal, and it is apparent in the lower figure that the tone-isolated signal did indeed contain fluctuations in amplitude. Frequency fluctuations were also present in the tone-isolated signal, but are not visible in Figure 6. Note also that only the first five seconds of the total 69.7 second time history are shown for clarity. 

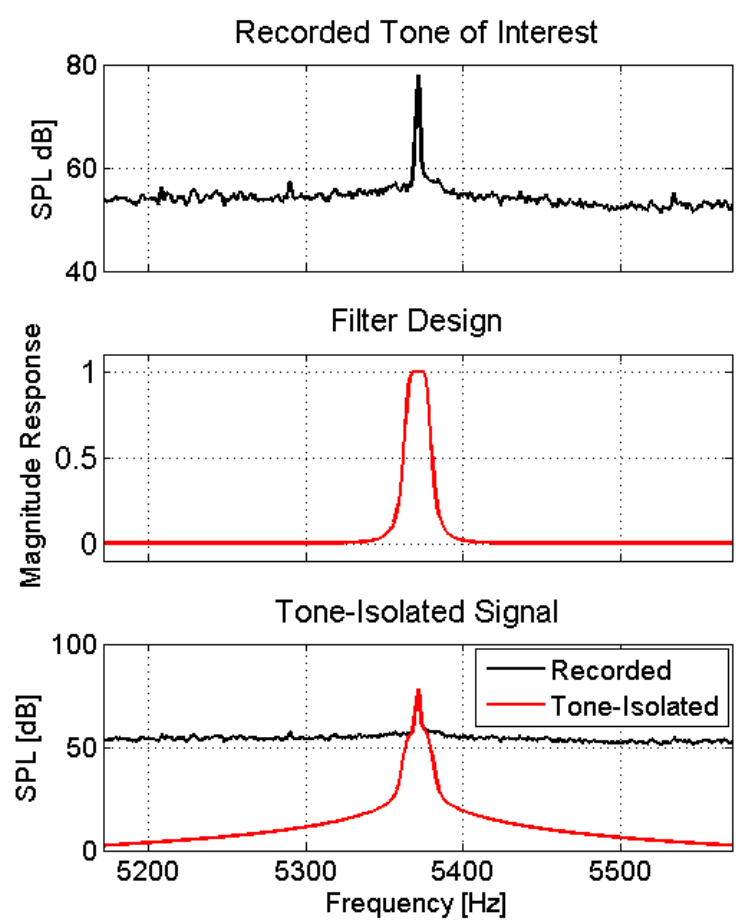

Figure 5. Recorded spectrum, filter design, and output spectrum from tone isolation process $(48 \%$ engine power, $30^{\circ}$ emission angle, $3 B P F$ tone $\left(f_{\text {mean }}=5,370 \mathrm{~Hz}\right)$ ).

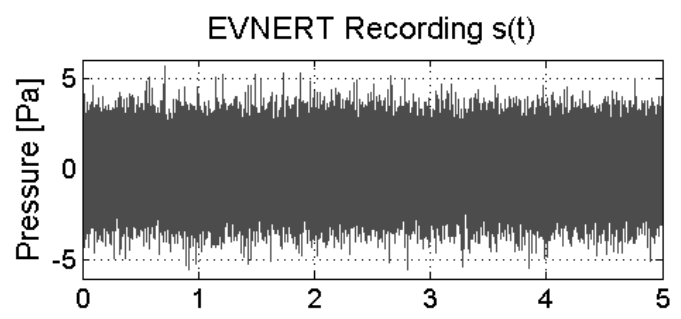

Tone Isolated, $s_{k}(t)$

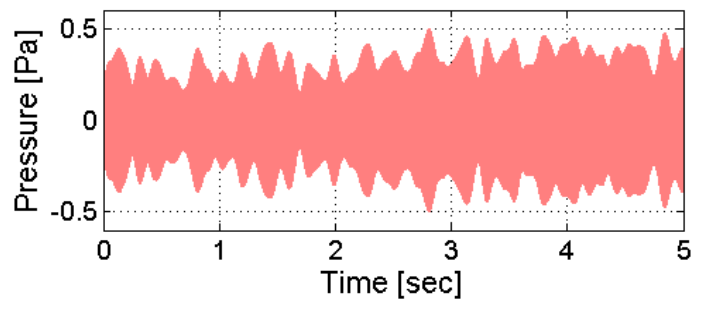

Figure 6. Pressure-time histories of the original recorded and tone-isolated signals for the $3 B P F$ tone $\left(f_{\text {mean }}=5,370 \mathrm{~Hz}\right)$ at $48 \%$ engine power and $30^{\circ}$ emission angle.

The MATLAB command hilbert( $)^{8}$ was used to generate the analytic signal, using removal of negative frequency information in the DFT as described earlier. The discrete-time amplitude envelope was then obtained by the magnitude of the tone-isolated analytic signal. Each envelope extracted from a tone of interest was then shifted to zero-mean by removing the time averaged value over the 69.7 second recording. Figure 7 shows the resulting amplitude fluctuation $a_{m o d, k}$ which was obtained from the envelope of the analytic signal. The standard deviation of the observed fluctuation in tonal amplitude was $0.0964 \mathrm{~Pa}$, which was about $45 \%$ of the mean observed amplitude in that tone. One such amplitude fluctuation was measured for each tone of interest. The standard deviations of amplitude fluctuations observed across the analyzed EVNERT data set were almost always less than $60 \%$ of the mean tonal amplitude.

Just as amplitude modulations were calculated from the analytic signal, frequency modulations were also obtained from the same MATLAB tool. The plot in Figure 8 shows an example of one such modulation, for the fan $\mathrm{BPF}$ tone as measured in the recording made at $54 \%$ power setting and $40^{\circ}$ emission angle. The BPF tone in that recording was well above the estimated broadband noise floor. Departures from the mean frequency varied by tone, emission angle, and power setting, but in general were relatively small. Typical frequency departures observed were almost always less than $0.5 \%$ of the mean frequency. 


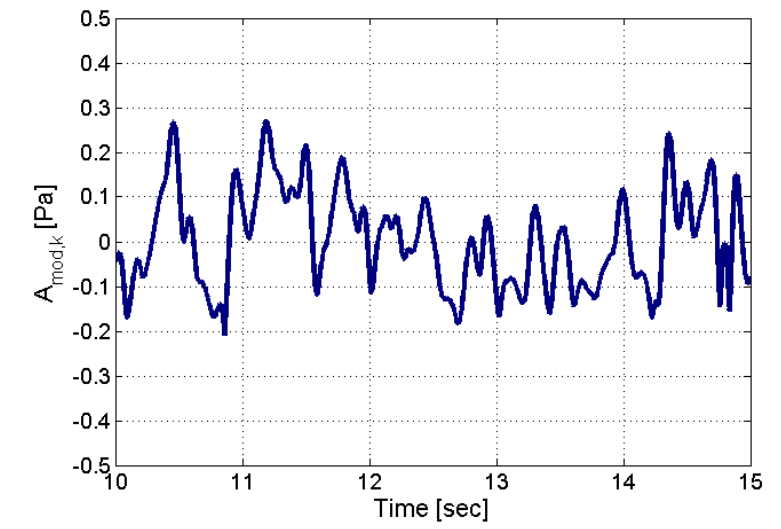

Figure 7. Extracted zero-mean amplitude fluctuation of the BPF tone at $60 \%$ engine power setting and $30^{\circ}$ emission angle.

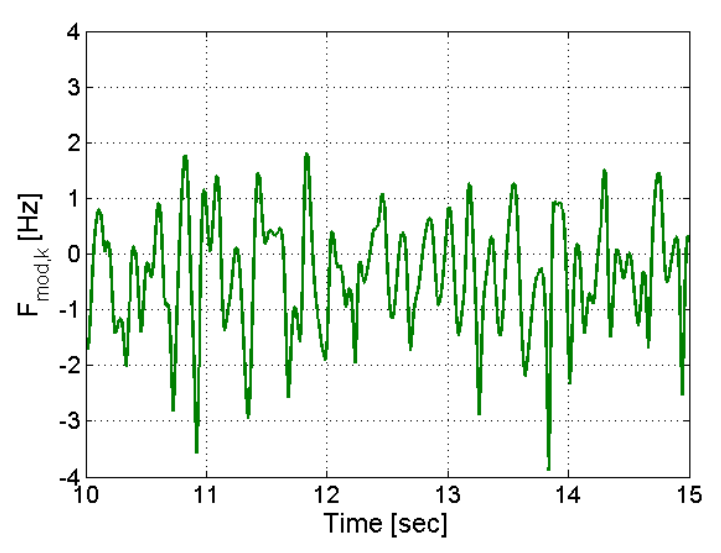

Figure 8. Extracted zero-mean frequency fluctuation in the fan BPF tone in the recording made at $54 \%$ power setting and $40^{\circ}$ emission angle.

\section{Characterization of Fluctuations}

A model was then created to characterize measured fluctuations and allow the synthesis of new fluctuations which are perceptually similar to those measured. The number of parameters and computational cost must be low, since random fluctuations must be generated for amplitude and frequency of about 100 tones when MPT noise is present. Additionally, the final model must be able to provide predictions for values where parameters were not directly measured to support the practical uses of synthesized noise, as described later.

Psychoacoustic literature notes that humans tend to discriminate fluctuations based on two criteria; the rate (speed) of modulation, and the modulation depth ${ }^{2}$. Those two parameters made natural choices for a model of tonal fluctuations designed to match human perception. Inspection of the amplitude fluctuation (e.g. Figure 7) indicates that they contain primarily low frequency components, but with no discernible periodicity. With these ideas in mind, the model chosen for tonal fluctuations was that of a bandlimited random process with a specified power spectral density (PSD). Two parameters characterized each PSD estimate, and corresponded directly to the psychoacoustic parameters noted. The first PSD parameter was the cutoff frequency of a certain fitted low-pass filter shape (developed in the next section), and the second was the variance of the fluctuation.

The bandwidth of spectral energy in a given fluctuation was estimated by calculating the cutoff frequency of a fitted filter. This process required several steps. The power spectral density of the zero-mean fluctuation was first calculated. Next, the maximum value of the PSD was obtained as a rough estimate for the passband gain of the filter. A weighting vector was prepared to exclude PSD bins with insignificant energy content from the fit routine. A $3^{\text {rd }}-$ order Butterworth filter magnitude response curve was chosen as the prototype for the spectral shape of the model. The weighted root-mean-square (RMS) deviation between the PSD of the measured fluctuation and the prototype filter was minimized as a function of the passband gain and cutoff (half-power) frequency. The result of the nonlinear fit routine was the cutoff frequency of a filter with minimum RMS error from the measured power spectral density. A graphic which displays a measured PSD and the resulting fit for the frequency fluctuation in the 1BPF tone at $48 \%$ power and $75^{\circ}$ emission angle is shown in Figure 9. The original measured PSD is shown in solid green, while the fitted filter response is shown in dashed red. The filter matches the general trend in the measured fluctuation, up to the limit of the weighted region near $30 \mathrm{~Hz}$. The cutoff frequency of the fitted filter (shown in pink), along with the calculated variance of the fluctuation, were then stored along with all other measurements.

The result of modeling each fluctuation as a bandlimited random process resulted in the characterization of amplitude and frequency modulations for each tone in each microphone recording at each power setting. The total number of parameters stored was 24,064 - bandwidth and variance for the amplitude and frequency fluctuations of each of the 6,016 target tones. Each set of parameters could be visualized as a surface for each tone, with measured values linearly interpolated as a function of engine power setting and emission angle. 
Two dimensional linear interpolation on each parameter resulted in four fit surfaces for each of the 88 tones analyzed. Figure 10 shows the bandwidth of amplitude fluctuations extracted from the 2BPF tone as measured in all analyzed EVNERT data in units of Hz. Figure 11 shows the variance of amplitude fluctuations in units of $\mathrm{Pa}^{2}$. Figure 12 and Figure 13 show the corresponding model parameters for frequency fluctuations as measured for the same tone. Some lobed behavior can be observed in each parameter in emission angles from $10^{\circ}$ to $50^{\circ}$ off-axis. Since measured tonal noise was not dominant at aft radiation angles, tonal parameters are less prominent.

Interpolated surfaces generated for each tone could then be probed to obtain an estimate of the fluctuation parameter values at a point where no recording of the TECH977 engine was made. Inside of the measured regions for power setting and emission angle, linear interpolations were used as estimates between measurement points. Outside of the regions, the nearest measured value was used.

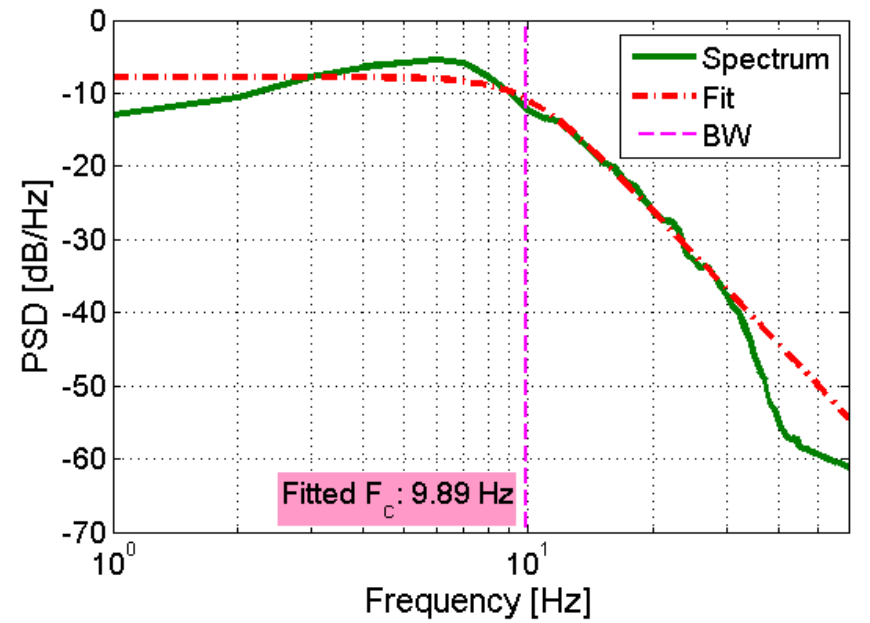

Figure 9. Results of the RMS deviation minimization for frequency fluctuations in the $1 \mathrm{BPF}$ tone at $48 \%$ engine power and $75^{\circ}$ emission angle.

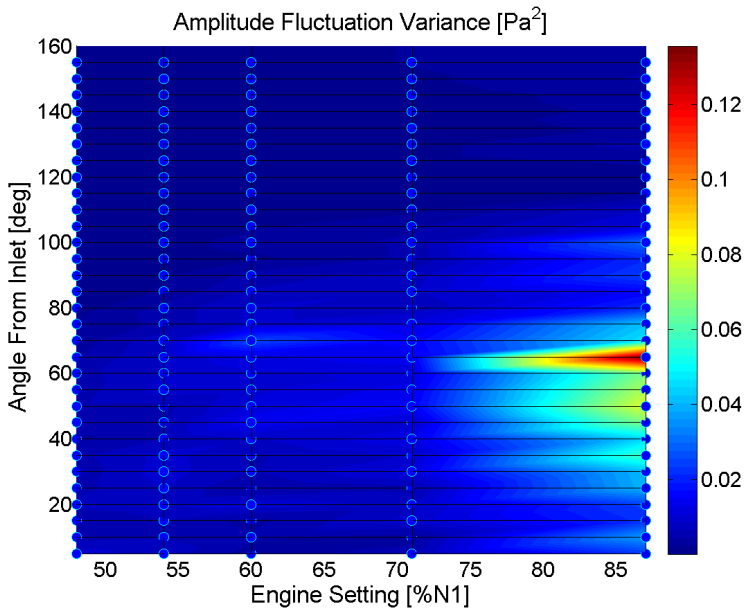

Figure 11. Interpolated surface for variance $\left[\mathrm{Pa}^{2}\right]$ of amplitude fluctuations in the $2 B P F$ tone in analyzed EVNERT recordings.
Figure 10. Interpolated surface for bandwidth [Hz] of amplitude fluctuations in the 2BPF tone in analyzed EVNERT recordings.

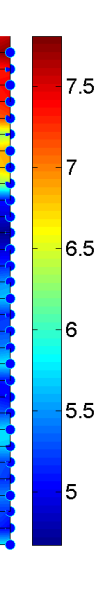

9

American Institute of Aeronautics and Astronautics 


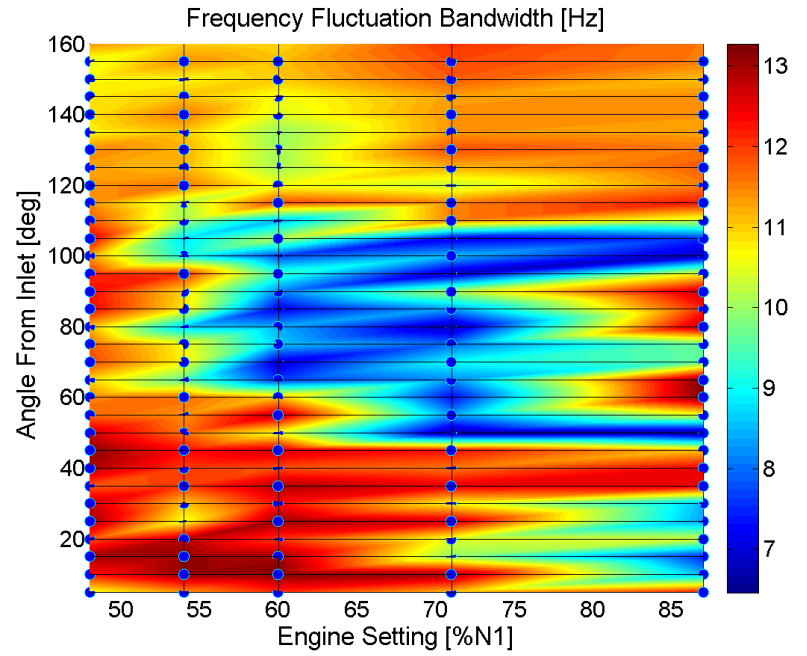

Figure 12. Interpolated surface for bandwidth $[\mathrm{Hz}]$ of frequency fluctuations in the $2 \mathrm{BPF}$ tone in analyzed EVNERT recordings.

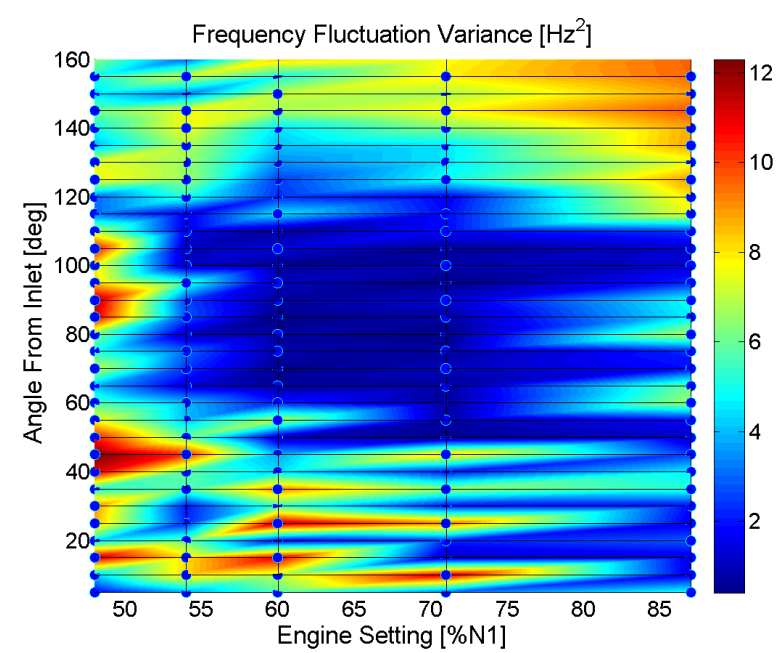

Figure 13. Interpolated surface for variance $\left[\mathrm{Hz}^{2}\right]$ of frequency fluctuations in the $2 \mathrm{BPF}$ tone in analyzed EVNERT recordings.

\section{Synthesis Procedures}

Broadband and tonal noise sources were synthesized separately using different methods. The broadband noise source $b(t)$ was synthesized using a moving synthesis buffer with time-variant one-third-octave band SPL specified at each synthesis block. That method can be viewed as the inverse operation of the STFT analysis method used to characterize the broadband noise $\mathrm{e}^{1,4}$, and it is based on overlap-add methods often used in audio synthesis ${ }^{9}$.

For the tonal source, multi-tone synthesis was performed by directly summing many individual tones in discrete time as

$$
\hat{s}[n]=\sum_{k=1}^{K} \hat{s}_{k}[n]
$$

where 'hats' denote synthesized pressure-time histories. Integration of instantaneous frequency to obtain the cosine argument was approximated during synthesis in the discrete time domain for the single-tone model. Specifically, each tone was generated by providing the amplitude envelope and instantaneous frequency $\hat{f}_{k}$ directly to the singletone model, as shown by

$$
\hat{s}_{k}[n]=\hat{a}_{k}[n] \cos \left(\frac{2 \pi}{F_{s}} \sum_{i=1}^{n} \hat{f}_{k}[i]+\hat{\phi}_{0, k}\right) .
$$

Note the inclusion of the constant $\phi_{0, k}$ as the last argument of the cosine function, corresponding to the constant of integration for instantaneous frequency. For cases where it was desired to directly synthesize an observed set of tonal fluctuations, that constant phase offset was important to ensure time alignment of the synthesis with the original measurement. When synthesizing tonal noise which was not supposed to mimic some measured set of fluctuations, the constant phase offset was set to random in the interval $[-\pi, \pi]$.

Since mean values and fluctuations are specified separately in the single-tone model, they may be obtained from different sources. Generally, mean values for fan noise synthesis are provided by an engine noise prediction tool, e.g. the Aircraft Noise Prediction Program (ANOPP) ${ }^{10,11}$. They may also be provided directly from measurement to ensure a match between time-averaged spectra, while at the same time providing simulated fluctuations around those measured means.

Figure 14 shows original measured fluctuations in amplitude and frequency for an example tone, compared with those fluctuations as obtained from analysis of a directly resynthesized noise. The comparison showed that the tonal synthesis routine generated a synthesized set of fluctuations which were in good agreement with the specific set provided. Some small differences are due to tonal energy leakage outside the analysis isolation filter window. 
However, the main objective of the work was to synthesize new tonal noise based on the parametric fluctuation model developed earlier, rather than directly synthesizing measurements. Modeled tonal fluctuations were generated by evaluating one realization of the corresponding bandlimited random process. The broadband synthesis tool was used to obtain that realization, but rather than specifying time-variant one-third-octave band levels as input, narrowband spectra were directly provided at each synthesis block. Those narrowband spectra were simply the prototype spectral shape evaluated with the appropriate bandwidth and variance. Note that specification of different fluctuation spectra at each synthesis block allowed model parameters to vary with time. That is, synthesis of tonal fluctuations could be performed for non-stationary bandwidth and variance. The capability to vary fluctuation model parameters as well as mean tonal amplitude and frequency proved to be useful for the ultimate goal of synthesizing realistic tonal noise in the context of an aircraft flyover.

One possible operation of interest when synthesizing tonal noise is that of an engine "spool-up," consisting of a continuous increase in power setting as might be encountered before a takeoff. During a spool-up, mean frequencies must increase while maintaining their harmonic relationship. MPT noise must also be carefully accounted for, as it cuts on once the fan reaches a supersonic condition. Figure 15 shows a simulated TECH977 spool up at a constant emission angle of $45^{\circ}$ which accounts for both characteristics. The engine power setting changes linearly between measured points, and once MPT noise was measured in the spectrum, it begins to cut on in the simulation as well (near 5 seconds).

Another practical operation of interest is to gradually change the emission angle at which source noise is synthesized. This operation is necessary to synthesize source noise corresponding to a continuously variant emission angle as needed for flyover simulation ${ }^{12,13}$. At low power, this operation would correspond to physically walking around the TECH977 engine from inlet to discharge while keeping power
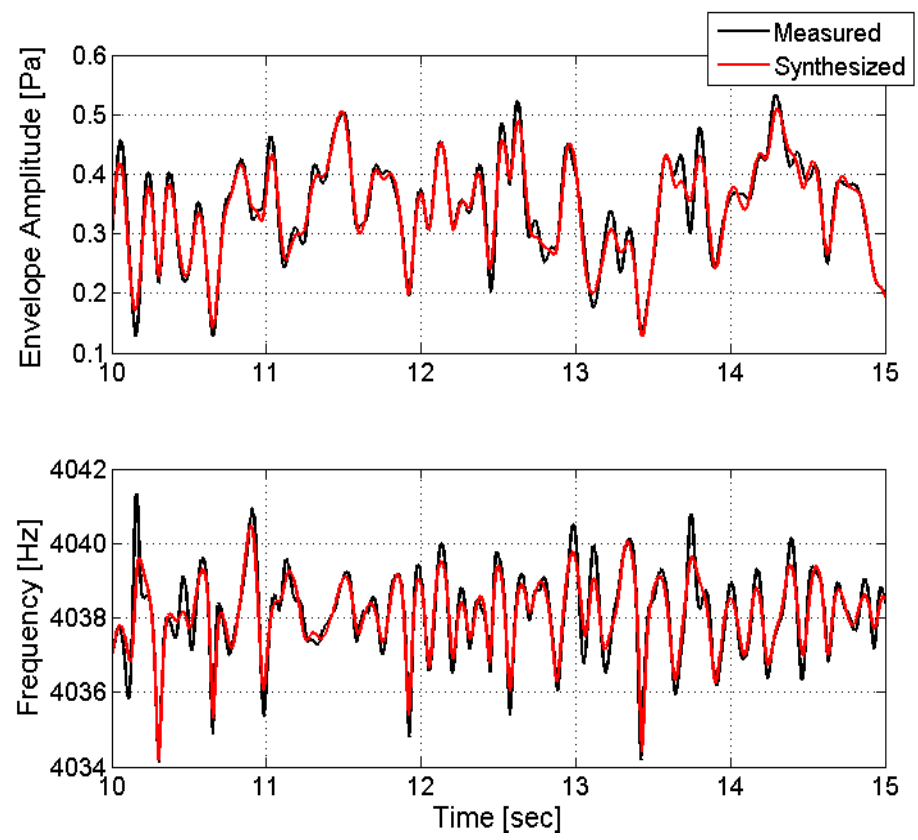

Figure 14. Comparison of measured and directly synthesized time histories for amplitude and frequency of the $2 \mathrm{BPF}$ tone at $48 \%$ engine power and $75^{\circ}$ emission angle.

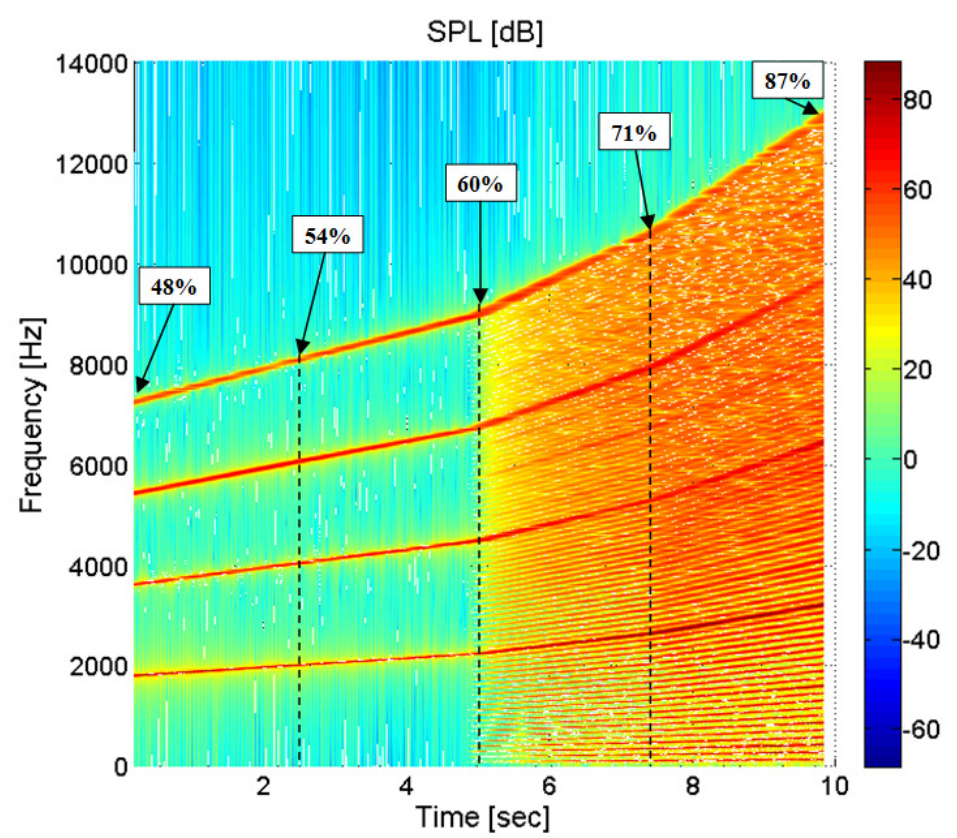

Figure 15. Synthesized engine spool-up at $45^{\circ}$ emission angle using timevariant mean values and fluctuations based on interpolated model parameters. 
setting constant at an idle condition of $48 \%$. Figure 16 shows the mean SPL of four synthesized multiples of the BPF versus time at the various input waypoints, which change level according to different regions of measured source directivity. Those waypoints are then interpolated at intermediate directivity angles during synthesis. Variance and bandwidth of fluctuations also change with respect to time. A spectrogram of the noise corresponding to this emission angle change can be seen in Figure 17, with SPL changes corresponding to those measured in EVNERT data.

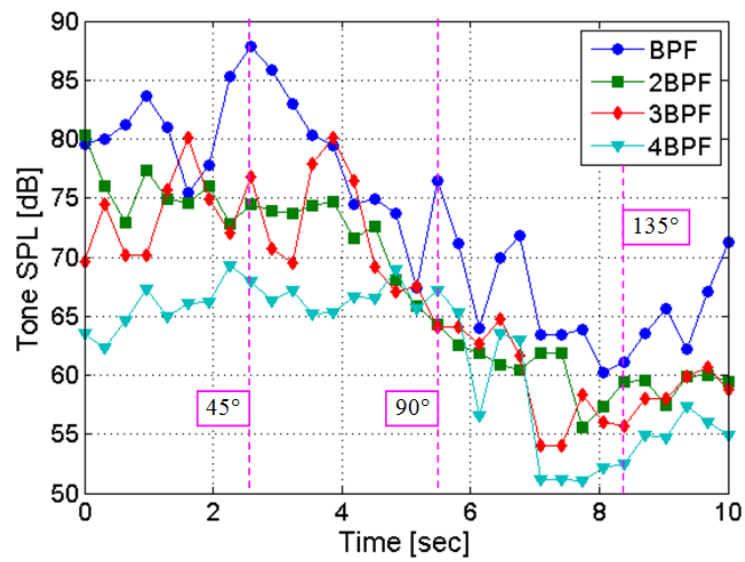

Figure 16. Mean SPL of four BPF multiples vs. time during simulated emission angle change at low engine power.

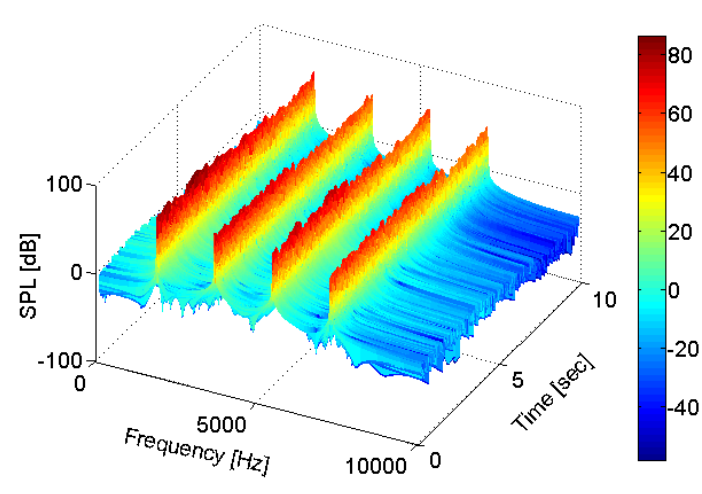

Figure 17. Simulated TECH977 emission angle variation from $5^{\circ}$ to $160^{\circ}$ off-axis over 10 seconds using time-variant fluctuation parameters.

\section{Subjective Listening Tests}

As presented in previous sections, quantitative comparisons showed good agreement between recorded and synthesized fan source noise. A subjective listening test was conducted to assess the perceived similarity between synthesized and recorded fan noise. The following section describes the experimental design and results of subjective listening tests.

\section{A. Experimental Design}

For the average listener, realism of fan source noise is difficult to evaluate with no reference for comparison; typical experiences are with complete flyover events which include all aircraft noise sources and propagation effects rather than source noise in isolation. Therefore, subjects were presented with paired noises and then asked to rate the degree of similarity between them. Listeners were informed that the sounds they were hearing were aircraft noises, but were not informed that at least one noise was always a recording. When comparing a synthesized noise source with a recorded noise source, a high similarity rating was understood to mean that "the synthesized source sounds like a real source." In this way, a similarity score was used to imply the realism of synthesized fan noise rather than attempting to measure realism directly.

It was hypothesized that including fluctuations would increase the perceived realism of synthesized noise. The lowest similarity score was thus expected for the time-invariant simulation methods, which did not include fluctuations in either the tonal or broadband source. Twenty comparisons were identified, and were divided into three categories, each with a specific purpose; they are referred to as "time-variant," baseline," or "time-invariant," as described below. Each category contained noises at two power settings (48 and $87 \%$ ) to evaluate synthesis performance for both BPF and MPT sources. Each category also contained two types of spectral content; tones in isolation and tones with the broadband noise source added. For cases where recorded tonal noise alone was required, narrow isolation filters were applied to recordings to separate tones from broadband noise. All noise segments in all comparisons were high-pass filtered using a $1,000 \mathrm{~Hz}$ cutoff frequency to remove any influence of jet noise features which were dominant at low frequencies. The lowest BPF tone of interest was $1,790 \mathrm{~Hz}$ for the $48 \%$ power setting.

Comparisons in the first category were intended to evaluate the performance of time-variant fan noise. Twelve "time-variant" pairs were included, each consisting of one segment of a fan noise recording and one segment of 
synthesized noise whose tonal fluctuations were based on the model developed earlier. For the sounds that included broadband noise, one-third-octave band SPL fluctuations were synthesized as measured from EVNERT recordings.

Four "time-invariant" pairs of fan noise were included where each consisted of recorded noise paired with noise which was synthesized without fluctuations in either the tonal or broadband component. Results from the timeinvariant category were compared to those from the time-variant category to evaluate the gains made by including fluctuations.

Finally, four "baseline" comparisons were added. Baseline pairs were nominally identical, and consisted of two non-overlapping segments of the same recording. Thus, the addition of baseline pairs established a maximum expected score as a reference for similarity scores of time-variant and time-invariant pairs at the same conditions.

Thirty subjects were included in subjective evaluations. They were divided into five groups of six subjects each. Paired comparisons were presented synchronously over headphones to each group. Each pair was presented twice, in short succession, after which the subjects rated the similarity of the two sounds. A linear scale was used with verbal anchors ranging from "not at all" to "extremely." Numeric values from 1 to 5 were assigned for quantitative analysis. Additional details on the test setup may be found in ref. 4.

\section{B. Comparison Results}

Mean similarity scores for each category of comparisons are summarized in Table 1 . The results show that the mean similarity judgment was 3.8 for recorded tonal source noise compared to synthesized tonal noise without fluctuations. When fluctuations were added to synthesized tones, the similarity score increased to 4.4. The baseline score for tone-isolated recorded noise against another segment of the same recording was 4.5.

The bottom row of Table 1 describes mean similarity scores for comparisons when both the broadband noise and tonal components were included. The mean score for the time-invariant category of comparisons was 4.1 . When fluctuations in both tonal noise and one-third-octave band levels for broadband noise were included, the mean similarity score increased to 4.2. The baseline score for comparisons where a segment of recorded noise was compared to another segment of the same recording was 4.3.

Table 1. Summary of mean similarity scores for each comparison category

\begin{tabular}{|c|c|c|c|}
\hline Spectral Content & $\begin{array}{c}\text { Time-Invariant } \\
\text { (fluctuations not included) }\end{array}$ & $\begin{array}{c}\text { Time-Variant } \\
\text { (fluctuations included) }\end{array}$ & $\begin{array}{c}\text { Baseline Recordings } \\
\text { (nominally identical) }\end{array}$ \\
\hline Tonal Only & 3.8 & 4.4 & 4.5 \\
\hline Tonal + Broadband & 4.1 & 4.2 & 4.3 \\
\hline
\end{tabular}

Mean response scores did not give sufficient information to either describe the distribution of judgments or identify significant differences between them. An Analysis of Variance (ANOVA) investigation was then conducted to assess the significance of differences observed between baseline, time-invariant, and time-variant comparisons. Two separate one-way ANOVA tests were performed to compare judgments in one category against the sample of judgments in a different category with the same spectral characteristics. In the subjective assessments discussed here, a 95\% confidence threshold was used. Thus, when the ANOVA returned p-values less than 0.05, differences between the judgments of the categories were understood to be significant. Conversely, for p-values greater than 0.05 , the underlying populations were said to be not significantly different.

The results of pairwise comparisons conducted as part of these ANOVA tests are summarized in Table 2, where statistically significant differences are denoted in red text. ANOVA results show that there were significant differences in the sample means of time-invariant and time-variant pairs for tonal noise, as well as between timeinvariant and baseline pairs for tonal noise. Notably, there were no statistically significant differences between timevariant and baseline pairs for tonal noise, or between any two comparison categories when the broadband source was included. 
Table 2. Results of several one-way ANOVA analyses performed on the results of subjective evaluations.

\begin{tabular}{|c|c|c|c|}
\hline \multirow{2}{*}{ Spectral Content } & \multicolumn{2}{|c|}{ Pairwise Comparisons } & \multirow{2}{*}{ P-value } \\
\hline & Category 1 & Category 2 & \\
\hline \multirow{3}{*}{ Tonal Only } & Time Invariant & Time Variant & 0.006 \\
\hline & Time Variant & $\begin{array}{c}\text { Baseline } \\
\text { Recordings }\end{array}$ & 0.23 \\
\hline & Time Invariant & $\begin{array}{c}\text { Baseline } \\
\text { Recordings }\end{array}$ & 0.004 \\
\hline \multirow{3}{*}{$\begin{array}{c}\text { Tonal + } \\
\text { Broadband }\end{array}$} & Time Invariant & Time Variant & 1 \\
\hline & Time Variant & $\begin{array}{c}\text { Baseline } \\
\text { Recordings }\end{array}$ & 0.08 \\
\hline & Time Invariant & $\begin{array}{c}\text { Baseline } \\
\text { Recordings }\end{array}$ & 0.5 \\
\hline
\end{tabular}

The scores for time-invariant tonal noise were significantly lower than those for both the time-variant $(\mathrm{p}=0.006)$ and baseline categories $(\mathrm{p}=0.004)$, indicating the importance of including fluctuations in synthesized sounds. The comparison of results for time-variant tonal noise against the baseline revealed that when modeled fluctuations in tonal amplitude and frequency were added, the resulting noise was not significantly different from recordings $(\mathrm{p}=0.23)$.

When broadband noise was added, no significant differences were found between scores for time-invariant noise and time-variant noise $(\mathrm{p}=1)$ or between time-invariant noise and the baseline comparisons $(\mathrm{p}=0.08)$. In other words, the improvement in perceptual realism using the new time-variant tonal synthesis method is reduced by the inclusion of a time-variant broadband noise component.

The change in similarity ratings with the addition of broadband noise could be explained in several ways. The total fan noise source is complicated, so subjects could have been preoccupied with cues from broadband and tonal sources and didn't discriminate time variations in either component. Perhaps the broadband component masked fluctuations in tonal amplitudes or frequencies. Research is available to quantify the just-noticeable-differences (JND) for pure amplitude or frequency modulated tones ${ }^{2}$. Measured amplitude fluctuations were generally above the applicable JND for a $1 \mathrm{kHz}$ pure tone, but the presence of the broadband noise source likely raised the threshold to differentiate time-invariant from time-variant noise. Perhaps amplitude fluctuations which were often above the JND in isolation might not have been perceptible in the presence of significant broadband noise. Additional work is required to determine the threshold of broadband noise below which time-variant syntheses can be differentiated from time-invariant syntheses. Results from this work could be used to justify inclusion or exclusion of time-domain fluctuations from future simulations, based on the relative contributions of tonal and broadband noise sources.

\section{Conclusion}

A set of turbofan engine recordings was used to extract time domain fluctuations for both tonal and broadband fan noise components. Broadband noise was characterized by fluctuations in one-third-octave band SPL. Each BPF or MPT tone of interest was isolated, and then used to construct the complex-valued analytic signal from which the amplitude envelope and instantaneous frequency were calculated. The analysis tools developed are applicable to general aircraft noise sources, whether dominated by tonal or broadband noise.

Extracted fluctuations in tonal parameters were modeled as bandlimited random processes, characterized by their variance and the cutoff frequency of a prototype filter which was fit to measured spectra. The model allows fluctuations to be estimated under engine operating conditions where measurements were not available, which in turn allows the synthesis of arbitrary variations in either power setting or emission angle. Synthesis tools were able to reproduce measured fluctuations in one-third-octave band SPL for broadband noise and modeled fluctuations in tonal noise. The synthesis tools developed do not operate in real-time, but should be easily modified to do so. Integration of time-varying synthesis methods into current NASA simulation tools ${ }^{12,13}$ is currently underway. Finally, subjective evaluations were conducted which implied that fluctuations in tonal parameters are an important discriminator for perceptual realism. Results demonstrated that inclusion of short-term fluctuations which were modeled and synthesized according to the methods developed here improved the perceived realism of the tonal noise source. However, that improvement is diminished by the inclusion of a broadband noise source. 


\section{Acknowledgements}

This work was supported by the Subsonic Fixed and Subsonic Rotary Wing Projects of the National Aeronautics and Space Administration (NASA) Fundamental Aeronautics Program, through a cooperative agreement (NNL09AA00A) with the National Institute of Aerospace (NIA) entitled "Analysis and synthesis of tonal noise sources for fixed and rotary wing aircraft" (NIA Activity Number 4740-009).

The authors would also like to thank Honeywell Aerospace for their role in producing the high quality data set required for this work.

\section{References}

${ }^{1}$ Grosveld, F.W., Sullivan, B.M., and Rizzi, S.A., "Temporal Characterization of Aircraft Noise Sources," Aerospace Sciences Meeting and Exhibit, Reno, Nevada, 2004.

${ }^{2}$ Zwicker, E. and Fastl, H. 2 ed, Psychoacoustics: Facts and Models. Springer-Verlag, Berlin and New York, 1999.

${ }^{3}$ Weir, D., ed., "Engine Validation of Noise and Emission Reduction Technology Phase I," NASA CR-2008-215225, 2008.

${ }^{4}$ Allen, M.P., "Analysis and Synthesis of Aircraft Engine Fan Noise for Use in Psyhoacoustic Studies," Master's Thesis, Mechanical Engineering, Virginia Polytechnic Institute and State University, Blacksburg, VA, 2012.

${ }^{5}$ Gabor, D., "Theory of Communication. Part 1: The analysis of information," Journal of the Institution of Electrical Engineers Part III: Radio and Communication Engineering, Vol. 93, No. 26, 1946, pp. 429-441.

${ }^{6}$ Boashash, B., "Estimating and Interpreting the Instantaneous Frequency of a Signal - Part 1: Fundamentals," Proceedings of the IEEE, Vol. 80, No. 4, 1991, pp. 520-538.

${ }^{7}$ Costain, J.K. and Coruh, C., Basic Theory of Exploration Seismology. Elsevier, Oxford, 2004.

${ }^{8}$ MathWorks, "Signal Processing Toolbox User's Guide," The Mathworks, Inc. Natick, MA 2012.

${ }^{9}$ Zolzer, U., ed., DAFX: Digital Audio Effects. John Wiley \& Sons, West Sussex, England, 2002.

${ }^{10}$ Kontos, K.B., Janardan, B.A., and Gliebe, P.R., "Improved NASA-ANOPP Noise Prediction Computer Code for Advanced Subsonic Propulsion Systems " NASA CR-195480, 1996.

${ }^{11}$ Hough, J.W. and Weir, D.S., "Aircraft Noise Prediction Program (ANOPP) Fan Noise Prediction for Small Engines," NASA CR-198300, 1996.

${ }^{12}$ Rizzi, S.A. and Sullivan, B.M., "Synthesis of Virtual Environments for Aircraft Community Noise Impact Studies," 11th AIAA/CEAS Aeroacoustics Conference, Monterey, CA, 2005.

${ }^{13}$ Rizzi, S.A., Sullivan, B.M., and Aumann, A.R., "Recent Developments in Aircraft Flyover Noise Simulation at NASA Langley Research Center," NATO RTA Applied Vehicle Technology Panel. AVT-158/ RSM-023, Montreal, Canada, 2008. 